\title{
Validación de la escala de catastrofismo ante el dolor (Pain Catastrophizing Scale) en deportistas españoles
}

\section{Validation of the Pain Catastrophizing Scale in Spanish athletes}

\author{
Validaçáo da escala de catastrofismo diante da dor (pain \\ catastrophizing scale) em esportistas espanhóis
}

\author{
Aurelio Olmedilla Zafra*, Enrique Ortega Toro*, Lucía Abenza Cano** \\ * Universidad de Murcia. ${ }^{* *}$ Universidad Católica San Antonio de Murcia (UCAM)
}

\begin{abstract}
Resumen: El objetivo del presente estudio consistió en validar la versión española de la Pain Catastrophizing Scale (PCS) de Sullivan, Bishop y Pivik (1995) en una muestra de 168 deportistas para la medida del catastrofismo ante el dolor. Se llevó a cabo la traducción de la escala y se estudiaron sus propiedades psicométricas. Los datos se sometieron a procedimientos de análisis factorial exploratorio. Los resultados indicaron que la escala presenta la misma estructura factorial de tres factores (rumiación, desesperación y magnificación) descritos en el estudio original, así como una adecuada consistencia interna (alfa de Cronbach $=0.818$ ). Los tres factores explican un total del $54.40 \%$ de la varianza. Los valores de consistencia interna de los factores se situaron entre 0.61 y 0.73 . Se concluye que la escala muestra propiedades psicométricas adecuadas por lo que su uso estaría indicado en la evaluación del catastrofismo, tanto para la práctica aplicada como para la investigación, en deportistas españoles.

Palabras clave: Catastrofismo, dolor, deportistas, validación.

Abstract: The goal of the present study was to validate the Spanish version of the Pain Catastrophizing Scale (PCS) by Sullivan, Bishop and Pivik (1995) in a sample of 168 athletes for measuring the amount of catastrophizing due to pain. The translation of the scale was done and the psychometric properties were studied. The data were subjected to procedures of exploratory factorial analysis. The results indicated that the scale presents the same three-factor factorial structure (rumination, helplessness, and
\end{abstract}

magnification) described in the original study, as well as adequate internal consistency (Cronbach's alpha $=0.818$ ). The three factors explain $54.40 \%$ of the variance. The values of internal consistency for the factors were between 0.61 and 0.73 . In conclusion, the scale demonstrates adequate psychometric properties so its use is indicated in the evaluation of catastrophizing, both for applied practice as well as research, in Spanish athletes.

Key Words: catastrophizing, pain, athletes, validation.

Resumo: O objetivo do presente estudo foi de validar a versão espanhola da escala de Pain Catastrophizing Scale (PCS) de Sullivan, Bishop y Pivik (1995), com uma mostra de 168 esportistas para a medida do catastrofismo diante da dor. Foi realizada a tradução da escala e estudadas as propriedades psicrométricas. Os dados foram sobmetidos a procedimentos de analises fatorial de três fatores (ruminação, desesperação e magnificação) descritos em um estudo original, assim como uma adequada consistência interna (alfa de Cronbach $=0.818$ ). Os três fatores explicam um total de $54.40 \%$ de variação. Os valores de consistência interna dos fatores situaram-se entre 0.61 e 0.73 . Conclui-se que a escala mostra propriedades psicrométricas adequadas, com isso seu ouso estaria indicado na avaliaçáo do catastrofismo, tanto para a pratica aplicada como para a pesquisa em esportistas espanhóis. Palavras chave: Catastrofismo, dor, esportistas, avaliaçấo.

\section{Introducción}

La lesión deportiva es un hecho traumático que, en función de su nivel de gravedad, puede suponer un punto de inflexión en la vida de cualquier deportista. Se podría definir como cualquier daño corporal, causado por una herida, golpe o enfermedad, cuyas consecuencias se vean reflejadas directamente en el rendimiento deportivo de la persona lesionada (Ramírez, Alzate y Lázaro, 2002). A veces conlleva un periodo de inactividad, que puede tener consecuencias adversas, tanto para él como para su entorno. Los factores personales y situacionales, tales como la gravedad de la lesión, el momento de la competición, el apoyo social o las habilidades de

Correspondencia: Aurelio Olmedilla Zafra. Facultad de Psicología Departamento de Personalidad, Evaluación y Tratamiento Psicológico. Correo electrónico: olmedilla@um.es afrontamiento del deportista, pueden mediatizar tales consecuencias e influir, por tanto, en su recuperación. De igual modo, la lesión puede afectar al rendimiento del deportista ya que, tal y como indica Buceta (1996) supone una disfunción en el organismo que provoca dolor, conlleva una interrupción o limitación de la actividad deportiva, así como de otras actividades extradeportivas, puede provocar cambios en la vida personal y familiar, la rehabilitación requiere tiempo, esfuerzo y dedicación y, en ocasiones resistencia al dolor y a la frustración; además, frecuentemente, trae consigo estados psicológicos que afectan el bienestar del deportista lesionado y de los que le rodean (irritabilidad general y estados depresivos, entre otros).

Además, la respuesta psicológica a la lesión provoca una reacción de pérdida con ciertos estados de ánimo, pensa- 
mientos y conductas que pueden agravar las dificultades propias de la recuperación (Tripp, Stanish, Ebel-Lam, Brewer y Birchard, 2011). También, se pueden considerar los factores personales y situacionales como variables que median en la respuesta a la lesión (Wiese-Bjornstal, Smith, Shaffer y Morrey, 1998). El dolor es una de las consecuencias inmediatas y duraderas de la experiencia de la lesión; para Wiese-Bjornstal (2002) el dolor se relaciona con la valoración cognitiva, la respuesta emocional y la respuesta conductual del deportista lesionado. Desde una perspectiva cognitiva, la forma en que los factores cognitivos se combinan o interactúan con factores sensoriales puede aproximarnos a la definición de dolor (Plata-Muñoz, Castillo-Olivares y Guevara-López, 2004). Los modelos de estrés y afrontamiento están siendo satisfactoriamente utilizados para explicar las diferencias en los niveles de ajuste entre los pacientes con dolor crónico (Jensen et al., 2002). En este sentido, el dolor es considerado como un importante agente estresante que es necesario afrontar eficazmente para, en la medida de lo posible, minimizar dicha experiencia (Plata-Muñoz et al., 2004). Por otro lado, el dolor es un aspecto central en la práctica de actividades físicodeportivas (Sullivan, Tripp, Rodgers y Stanish, 2000), y suele ser considerado como un obstáculo a la rehabilitación de la lesión (Heil, 1993).

Según Sullivan et al. (2001), en los últimos años la función del constructo catastrofismo ante el dolor en la modulación de las respuestas dolorosas ha recibido una considerable atención, demostrándose una relación coherente entre el catastrofismo y las reacciones de malestar ante estímulos dolorosos. En general, tal y como indican Sullivan, Bishop y Pivik (1995), la mayoría de autores están de acuerdo en que el término catastrofismo hace referencia a una percepción mental negativa y exagerada respecto a la experiencia de dolor, tanto real como anticipada. Aunque no están claras las causas del catastrofismo ante el dolor (García-Campayo et al., 2008), se ha demostrado que es crucial en su desarrollo un modelo de apego inseguro (McWilliams y Asmundson, 2007) y cierta sensibilidad ante la ansiedad (Reiss, 1991). Gran parte de la literatura científica sugiere que las principales consecuencias asociadas al catastrofismo son dolor más intenso, mayor consumo de analgésicos, disminución de las actividades diarias e incapacidad laboral (Sullivan et al., 2001); aunque también se ha demostrado mayor sensibilidad al dolor (Edwards, Fillingim, Maixner, Sigurdsson y Haythornthwaite, 2004), mayor depresión en el contexto del dolor crónico (Edwards, Clifton, Bingham, Bathon y Haythornthwaite, 2006), interferencias en el uso de estrategias de afrontamiento (Sullivan et al., 2001), menos adherencia a los tratamientos farmacológicos en enfermos reumáticos (Neame y Hammond, 2005), mayor actividad en las áreas cerebrales relacionadas con los procesos de dolor (atención, emoción y control motor) en pacientes de fibromialgia (Gracely et al., 2004), incremento de las conductas ante el dolor (Picavet, Viaeyen y Schouten, 2002), peor pronóstico (Stephens, Druley y Zautra, 2002), ideación suicida (Edwards, Smith, Kudel y Haythornthwaite, 2006) y periodos de rehabilitación más largos tras intervenciones quirúrgicas (Kendell, Saxby, Farrow y Naisby, 2001).

Sullivan et al. (2001) enmarcan las relaciones observadas entre catastrofismo y dolor dentro de un contexto teórico en el que el catastrofismo puede contribuir a que una experiencia de dolor sea más intensa aumentando el foco atencional en el dolor y/o aumentando la respuesta emocional ante los estímulos nocivos. Además, ese catastrofismo puede surgir como recurso de afrontamiento ante las emociones negativas causadas por el dolor crónico con la intención de reclutar apoyo social.

Varias investigaciones demuestran que los deportistas son más tolerantes al dolor y perciben menos intensidad del mismo que los sedentarios (Ahern y Lohr, 1997; Hamilton, Hamilton, Meitzer, Marshall y Molnar, 1989; Sullivan et al., 2000; Tajet- Foxell y Rose, 1995). Sin embargo, el catastrofismo es predictor de dolor tanto para los deportistas como para los sedentarios, explica las diferencias en la percepción de dolor entre hombres y mujeres, pero no explica/mediatiza las diferencias en la percepción de dolor entre deportistas y sedentarios (Sullivan et al., 2000). Encarnacion, Meyers, Ryan y Pease (2001), compararon las estrategias de afrontamiento ante el dolor de un grupo de bailarines profesionales frente a un grupo de deportistas y encontraron que los bailarines mostraban mayores niveles de catastrofismo que los deportistas. En esta misma línea, el trabajo de Paparizos, Tripp, Sullivan y Rubenstein (2004), relaciona catastrofismo y dolor, induciendo dolor a un grupo de bailarinas catalogadas en tres niveles (expertas, intermedias y principiantes), respecto a un grupo control. El catastrofismo estaba significativamente relacionado con el dolor (como predictor); las bailarinas más expertas mostraron más tolerancia al dolor que las principiantes; y las bailarinas en general toleraban mejor el dolor que el grupo control.

El trabajo de Tripp, Stanish, Reardon, Coady y Sullivan (2003) compara adolescentes y adultos lesionados del LCA, relacionando el dolor, el catastrofismo y la angustia afectiva (depresión y ansiedad) obteniendo mayores niveles de dolor, catastrofismo y ansiedad en los adolescentes. Meyers, Bourgeois y LeUnes (2001), descubrieron que los deportistas universitarios con más riesgo potencial de lesión (deportes de contacto) experimentaban valores menores de catastrofismo.

Según la revisión de Wiese-Bjornstal (2002), los deportistas que toleran mejor el dolor se recuperan más rápido; aquellos que adoptan una interpretación catastrofista obtienen menos éxitos en los protocolos de rehabilitación, que los que tienen una percepción positiva; y con respecto a las cogniciones, el miedo incrementa la tolerancia al dolor y la ansiedad la reduce. Estos resultados pueden entenderse si tenemos en cuenta la relación 
entre el dolor psicológico y la recuperación física. Por ejemplo, componentes del dolor psicológico como la depresión y la ansiedad aumentan la actividad del sistema nervioso autónomo (incremento de la tensión muscular, de la presión arterial, de la frecuencia cardiaca, de frecuencia respiratoria, etc.) y perjudican la función inmune que, a su vez puede, romper el proceso de recuperación física del deportista (Cramer y Perna, 2000).

Keefe, Rumble, Scipio, Giordano y Perri (2004) indican que hay razones consistentes que avalan el estudio del catastrofismo ante el dolor. Primero, encontramos varias medidas fiables y válidas del catastrofismo ante el dolor; la escala Pain Catastrophizing Scale (PCS) de Sullivan et al. (1995); la escala de catastrofismo del Coping Strategies Questionnaire (CSQ) (Rosenstiel y Keefe, 1983); la escala de catastrofismo del Cognitive Coping Strategy Inventory (CCSI) (Butler, Damarin, Beaulieu, Schwebel y Thorn, 1989) y la subescala de catastrofismo del Sport Inventory of Pain (SPI) de Meyers, Bourgeois, Stewart y LeUnes (1992). Segundo, los estudios se han dirigido a diferentes muestras clínicas de adultos y niños (Crombez, Vlaeyen, Heuts, Lysens, 1999) así como a la población general. Tercero, aunque el catastrofismo se relaciona con el dolor, continua siendo relacionado significativamente con otras medidas de ajuste para controlar el nivel de dolor. Cuarto, aunque se ha sugerido que los efectos del catastrofismo pueden ser debidos a la depresión subyacente, numerosos estudios (Geisser et al., 1994; Haaga, 1990; Jensen et al., 1991; Keefe et al., 2000; Sullivan, Stanish, Waite, Sullivan y Tripp, 1998) evidencian que el catastrofismo es predictor del dolor después incluso de controlar la depresión. Finalmente, hay evidencias de que el nivel de catastrofismo puede cambiar durante el transcurso de los programas de intervención psicológica, y de que esos cambios se relacionan a largo plazo con mejoras en el dolor, el estado psicológico y la capacidad física.

La investigación del catastrofismo ante el dolor también tiene algunas limitaciones. Primero, la mayor parte de los estudios son empíricos y no están basados en una teoría sistemática del catastrofismo al dolor. Sullivan et al. (2001) propusieron un modelo de catastrofismo al dolor realizando una aportación teórica de referencia en este campo. Segundo, existen muchas investigaciones transversales haciendo difícil determinar si el catastrofismo supone un mal ajuste al dolor para el sujeto o viceversa. Algunos estudios longitudinales sobre programas de intervención demuestran que si el catastrofismo desciende al principio del tratamiento, predice una disminución del dolor al final del mismo (Burns, Glenn, Bruehl, Harden y Lofland, 2003; Burns, Kubilus, Bruehl, Harden y Lofland, 2003). Algunos estudios realizados con población hispano parlante (Moral, González-Ramírez y Landero, 2011; Rodero, García Campayo, Casanueva y Sobradiel, 2008) han probado la eficacia del tratamiento cognitivo-conductual para el descenso del catastrofismo en enfermos de fibromialgia.
La escala Pain Catstrophizing Scale (PCS) es uno de los instrumentos más utilizados para medir el catastrofismo ante el dolor (Edwards et al., 2006; Sullivan et al., 1998) demostrando su validez y fiabilidad desde su origen (Severeijns, Van den Hout, Vlaeyen y Picavet, 2002; Sullivan et al., 1995; Van Damme, Crombez, Bijttebier, Goubert y Van Houdenhove, 2002). Además, la escala PCS ha servido como instrumento referente en investigaciones de todo el mundo siendo adaptada y validada en poblaciones diversas, como por ejemplo la versión para familiares de enfermos crónicos de Cano, Leonard y Franz (2005); la de Crombez et al. (2003) para nińos; la de Goubert et al. (2006) para los padres de niños enfermos; o la de Chibnall y Tait (2005) para trabajadores lesionados. También ha sido adaptada para diferentes culturas como la versión alemana de Meyer, Sport y Mannion (2008), la versión catalana de Miró, Nieto y Huguet (2008), la versión para adolescentes franceses de Tremblay et al. (2006), o la versión china de Yap et al. (2008). En España, la escala PCS ha sido escasamente empleada, pudiendo citarse como pionero el trabajo de García-Campayo et al. (2008) donde se valida la versión española en una muestra clínica de fibromialgia.

El catastrofismo ante el dolor, pese a su importancia, apenas se ha estudiado en deportistas con lesión (Wiese-Bjornstal, 2002). Por ello, la validación al castellano y la adaptación a la población deportista española de la referida escala es el principal objetivo de este estudio. Además, se pretende relacionar la escala con los niveles de depresión y ansiedad de los deportistas.

\section{Método}

\section{Participantes}

La muestra del estudio estuvo formada por 168 deportistas de competición, 146 de ellos eran hombres y 22 mujeres. Su edad estaba comprendida entre 14 y 53 ańos (con una media de 24.77 años y una desviación típica de 6.59 años) y la experiencia competitiva, respecto a la práctica de su deporte, era de 10.83 años de media (con una desviación típica de 5.94 años). Pertenecían a diferentes modalidades deportivas, principalmente de tipo colectivo, y la mayoría participaban en competiciones regionales y nacionales. Inicialmente, la escala fue completada por 78 deportistas sanos y, posteriormente, se administró a otros 90 deportistas afectados con lesiones de distinta consideración que recibían tratamiento de rehabilitación en un centro de fisioterapia.

\section{Instrumentos}

La PCS es una escala autoadministrada de 13 ítems y una de las más utilizadas para valorar el catastrofismo ante el dolor. En ella, los sujetos toman como referencia sus experiencias 
dolorosas pasadas e indican el grado en el cual experimentaban cada uno de los 13 pensamientos o sentimientos en una escala Líkert de 5 puntos que va de 0 (nunca) a 4 (siempre). De la escala se obtiene una puntuación total que refleja el nivel de catastrofismo ante el dolor del sujeto. Comprende 3 dimensiones: a) rumiación (la preocupación constante y la incapacidad de inhibir pensamientos relacionados con el dolor); b) magnificación (la exageración de lo desagradable de las situaciones de dolor y las expectativas de consecuencias negativas), y c) desesperación (la incapacidad frente a situaciones dolorosas). El intervalo teórico del instrumento se sitúa entre 13 y 62, indicando las puntuaciones bajas escaso catastrofismo, y los valores altos, elevado catastrofismo.

La depresión fue medida con el Inventario de Depresión de Beck (BDI; Beck, Ward, Mendelson, Mock y Erbaugh, 1961). El BDI ha sido utilizado para medir síntomas depresivos en poblaciones clínicas, incluyendo pacientes con dolor crónico y deportistas post-quirúrgicos (Tripp et al., 2003). El BDI es tan fiable (Beck et al., 1961; Beck, 1970) como válido (Bumberry, Oliver y McClure, 1978) y un instrumento de medida estándar para la depresión en la investigación médica y psicológica.

La ansiedad-rasgo fue medida con el Cuestionario de Ansiedad de Estado-Rasgo (STAI; Speilberger, Gorsuch y Lushene, 1970). El STAI tiene alta consistencia interna y validez (Spielberger, Gorsuch, Lushene, Vagg y Jacobs, 1983) y fue utilizado en uno de los estudios realizados por Sullivan et al. (1995) para el desarrollo de la PCS.

\section{Procedimiento}

Se obtuvo el permiso para traducir y validar la PCS de los autores originales (Sullivan et al., 1995). Se seleccionó a una traductora, profesional del área de la actividad física y del deporte, conocedora de ambas culturas y de los objetivos de la PCS que tradujo por primera vez la escala al espańol, adoptándose un diseño de traducción directa.

A continuación, esta primera adaptación al castellano de la PCS fue valorada, por separado, por siete psicólogos del deporte de reconocido prestigio. Cada psicólogo analizó la validez del contenido y comentaron individualmente las definiciones, dimensiones y enunciados de la versión española del cuestionario. Posteriormente, se realizó una síntesis de las valoraciones, de la que se obtuvo una versión renovada. Por último, se contrastó esta versión con la adaptación realizada por García Campayo et al. (2008), observándose su alto grado de coincidencia y obteniéndose al término del proceso la versión definitiva del instrumento.

La escala fue completada en primera instancia por deportistas no lesionados que competían en diferentes modalidades y categorías; posteriormente, se administró a deportistas que sufrían alguna lesión, inmersos en un programa de rehabilitación desarrollado por diferentes centros de fisioterapia con los que se había establecido previamente un acuerdo de colaboración. El consentimiento informado fue otorgado por todos los participantes del estudio.

\section{Resultados}

\section{Análisis Factorial de la Escala de Catastrofismo ante el Dolor para deportistas (ECD)}

La validez de esta escala se calculó a través de un análisis factorial mediante la extracción de componentes principales y rotación Varimax con Kaiser. Tanto la prueba KMO (.811), como la prueba de esfericidad de Barlett (sig. .000) mostraron buenos valores.

En la Tabla 1, previa eliminación de los factores con pesos inferiores a .05 , se puede apreciar que la concepción del catastrofismo ante el dolor se percibe desde tres dimensiones, por lo que los ítems se agrupan en tres factores principales y estadísticamente independientes. En concreto el factor 1 (rumiación), recibe las principales cargas de los ítems 8, 9, 10 y 11 referidos a la "preocupación constante". En el factor 2 (desesperación) cargan mayoritariamente los ítems 1, 2, $3,4,5$ y 12 , referidos a la "incapacidad frente a situaciones dolorosas". Finalmente, el factor 3 (magnificación) recibe las principales cargas de los ítems 6,7 y 13 , relacionados con la “exageración”.

Tabla 1. Análisis factorial de los ítems de la ECD para deportistas

\begin{tabular}{lccc}
\hline ÍteMS & $\begin{array}{c}\text { FACTOR I } \\
\text { RUMIACIÓN }\end{array}$ & $\begin{array}{c}\text { FACTOR 2 } \\
\text { DESESPERACIÓN }\end{array}$ & $\begin{array}{c}\text { FACTOR 3 } \\
\text { MAGNIFICACIÓN }\end{array}$ \\
\hline Ítem 9 &, 758 & & \\
Ítem 8 &, 684 & & \\
Ítem 11 &, 683 & & \\
Ítem 10 &, 673 & & \\
Ítem 4 & &, 794 & \\
Ítem 5 & &, 778 & \\
Ítem 12 & &, 610 & \\
Ítem 3 & &, 591 &
\end{tabular}




\begin{tabular}{lcc} 
Ítem 2 &, 544 & \\
Ítem 1 &, 501 & \\
Ítem 13 & &, 714 \\
Ítem 6 &, 625 \\
Ítem 7 &, 540 \\
\hline Porcentaje de la Varianza explicado 31,998 & 13,099 & 9,304 \\
\hline $\begin{array}{l}\text { Método de Extracción: Análisis de componentes principales. } \\
\text { Método de rotación: Normalización Varimax con Kaiser. }\end{array}$ \\
\hline
\end{tabular}

En la Tabla 1 se observa la varianza total explicada, de manera que los tres factores dan cuenta de un $54.40 \%$, porcentaje de explicación que se sitúa en niveles de aceptación elevados. En concreto el factor rumiación explica un $31.99 \%$ de la varianza, el factor desesperación un $13.09 \%$ y el factor magnificación un $9.30 \%$.

\section{Análisis de la fiabilidad de la Escala de Catastrofismo ante el Dolor para deportistas (ECD)}

La fiabilidad de la ECD se calculó mediante el coeficiente alfa de Cronbach, el cual debe interpretarse como un indi- cador de la consistencia interna de los ítems ya que se calcula a partir de la covarianza entre ellos. El valor obtenido de la ECD fue de Alfa $=0.818$. La subescala rumiación obtuvo un alfa $=.735$, la subescala desesperación obtuvo un alfa $=.737$, y finalmente la subescala magnificación obtuvo un alfa =.618.

En la tabla 2 se aprecian las medias y las varianzas de los 13 ítems de la ECD, así como el coeficiente alfa de Cronbach si se eliminara cada uno de los ítems; en este caso, se aprecia la necesidad de cada uno de ellos, puesto que ninguna eliminación supone un incremento sobre el alfa del cuestionario.

Tabla 2. Fiabilidad y valores estadísticos de la ECD para deportistas.

\begin{tabular}{|c|c|c|c|c|}
\hline FACTOR & ÍTEMS & Media & Desviación típica & Alfa de Cronbach si se elimina el elemento \\
\hline \multirow{5}{*}{ RUMIACIÓN } & Ítem 8 & 3,26 & 1,068 & ,704 \\
\hline & Ítem 9 & 1,76 & 1,035 & ,631 \\
\hline & Ítem 10 & 1,73 & ,938 & ,705 \\
\hline & Ítem 11 & 2,56 & 1,197 & ,652 \\
\hline & RUMIACIÓN & 9,30 & 3,17 &, 735 \\
\hline \multirow{7}{*}{ DESESPERACIÓN } & Ítem 1 & 2,20 & 1,117 & ,737 \\
\hline & Ítem 2 & 1,43 & ,994 & ,696 \\
\hline & Ítem 3 & 1,07 & 945 & ,694 \\
\hline & Ítem 4 & 1,19 & ,902 & ,695 \\
\hline & Ítem 5 & 1,09 &, 881 & ,698 \\
\hline & Ítem 12 & 1,71 & 1,005 & ,738 \\
\hline & DESESPERACIÓN & 8,68 & 3,89 & ,746 \\
\hline \multirow{4}{*}{ MAGNIFICACIÓN } & Ítem 6 & 1,89 & 1,038 & ,473 \\
\hline & Ítem 7 & 1,45 & 1,167 &, 565 \\
\hline & Ítem 13 & 1,68 & 1,073 &, 523 \\
\hline & MAGNIFICACIÓN & 5,02 & 2,47 & ,618 \\
\hline TOTAL & CATASTROFISMO & 23,02 & 7,49 & .818 \\
\hline
\end{tabular}

En la Tabla 2 se observa la media de los ítems pertenecientes a cada uno de los factores. Al analizar la relación entre las tres subescalas, los datos del presente estudio indican que, a pesar de apreciarse correlaciones estadísticamente significativas, el valor de la correlación es extremadamente bajo. Entre desesperación y magnificación $(\mathrm{r}=.383 ; \mathrm{p}<0.01)$, entre desesperación y rumiación $(\mathrm{r}=.433, \mathrm{p}<0.01)$, y entre magnificación y rumiación $(r=.430 ; p<0.01)$. Finalmente, al analizar la relación de las tres subescalas con la ECD global se observan relaciones estadísticamente significativas. Así ocurre entre 
desesperación y catastrofismo $(\mathrm{r}=.829 ; \mathrm{p}<0.01)$, entre magnificación y catastrofismo(r=.711; $\mathrm{p}<0.01)$, y entre catastrofismo y rumiación $(r=.790 ; \mathrm{p}<0.01)$.

\section{Análisis descriptivo de la Escala de Catastrofismo ante el Dolor para deportistas (ECD)}

Desde una perspectiva general, los datos reflejan que el deportista dispone de una media de 23.02 puntos en el cues- tionario de catastrofismo ante el dolor, con una desviación típica de 7,49 puntos. En la Figura 1 se aprecia que el factor que alcanza puntuaciones medias más elevadas es el factor rumiación (9.30), siendo el de magnificación el que dispone de los valores medios más bajos (5.02). El factor desesperación alcanza una media de 8.68. De igual modo se aprecia que los tres factores que componen la escala de catastrofismo presentan una desviación típica elevada, lo que supone la heterogeneidad de la muestra en estos factores.

Figura 1. Gráfica de Cajas de los tres factores de la ECD para deportistas.

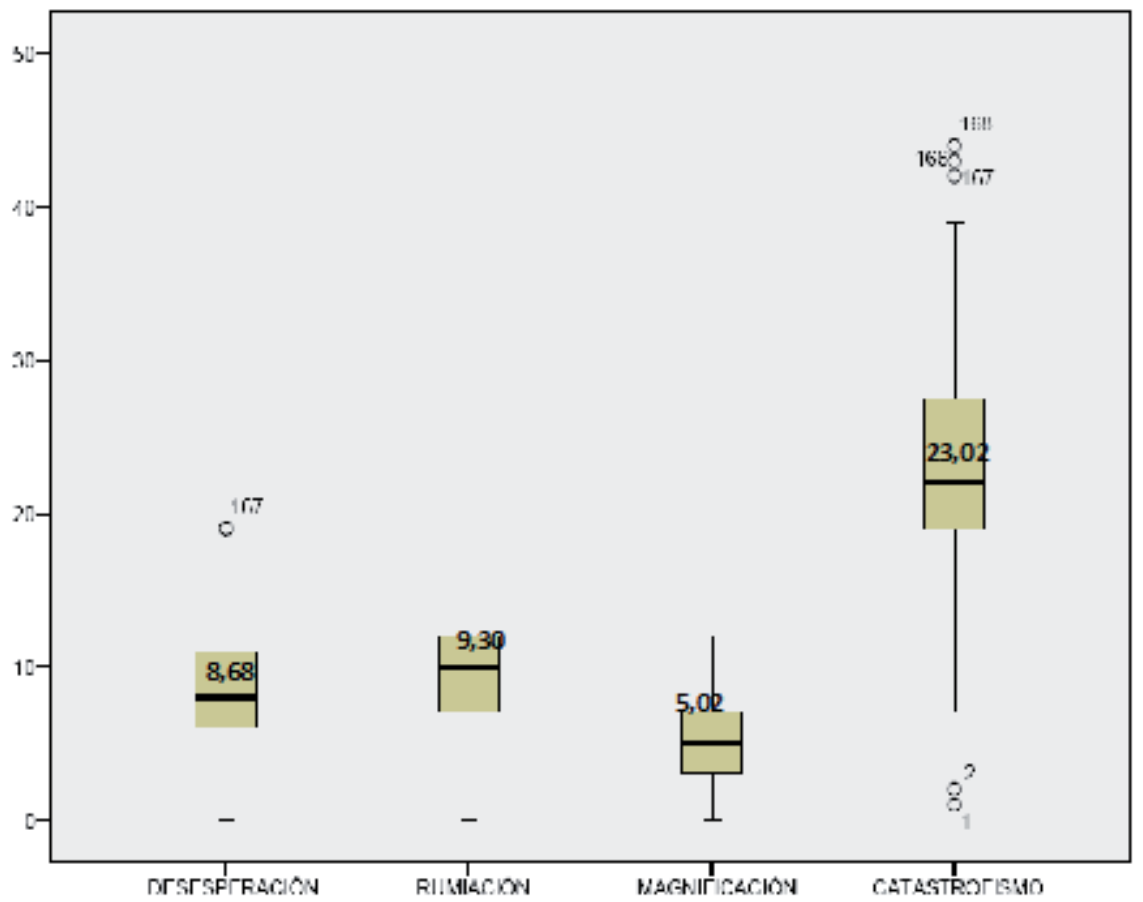

\section{Validez convergente}

Con el objetivo de apreciar las diferencias entre los deportistas lesionados frente a los no lesionados, se agruparon a los sujetos que conforman la muestra de deportistas en dos grupos; lesionados $(\mathrm{n}=78)$ y no lesionados $(\mathrm{n}=90)$. Se midió la relación de la ECD con la ansiedad rasgo (medida con el STAI-R) y la depresión (evaluada con el BDI). En el grupo de los deportistas no lesionados, la ECD global y sus subescalas no correlacionan ni con la ansiedad rasgo ni con la depresión. Es decir, no se observa relación entre rumiación y ansiedad rasgo $(\mathrm{r}=-.106 ; \mathrm{p}=.353)$ ni entre rumiación y depresión ( $\mathrm{r}=-$ .133 ; $\mathrm{p}=.245)$; tampoco se aprecia relación entre desespera- ción y ansiedad rasgo ( $\mathrm{r}=-.009 ; \mathrm{p}=.938)$ ni entre desesperación y depresión ( $\mathrm{r}=-.101 ; \mathrm{p}=.380)$; lo mismo ocurre si se analiza la relación entre magnificación y ansiedad rasgo ( $\mathrm{r}=.144$; $\mathrm{p}=.208$ ) o entre magnificación y depresión ( $\mathrm{r}=-.044 ; \mathrm{p}=.699)$; finalmente tampoco se observa correlación entre el catastrofismo ante el dolor y la ansiedad rasgo $(r=-.006 ; \mathrm{p}=.956) \mathrm{ni}$ entre el catastrofismo y la depresión ( $\mathrm{r}=-.126 ; \mathrm{p}=273)$.

Sin embargo, en el grupo de los deportistas lesionados se aprecian relaciones estadísticas significativas entre la escala global ECD y la ansiedad rasgo $(\mathrm{r}=.338 ; \mathrm{p}<0.01)$, así como entre cada unas de las subescalas, la rumiación $(\mathrm{r}=.208 ; \mathrm{p}<0.05)$, la magnificación $(\mathrm{r}=.240 ; \mathrm{p}<0.05)$ y la desesperación $(\mathrm{r}=.321$; $\mathrm{p}<0.01)$ con la ansiedad rasgo. Al analizar la relación entre 
ECD y la depresión, solo se aprecian relaciones estadísticamente significativas con la subescala desesperación $(\mathrm{r}=.261$; $\mathrm{p}<0.05)$.

\section{Discusión y conclusiones}

El propósito de este estudio consistió en validar la escala PCS para evaluar el catastrofismo ante el dolor en deportistas españoles. Comenzó con la caracterización de la lesión como hecho relevante en la práctica deportiva, y la percepción de dolor como inherente a aquella, así como la definición del constructo catastrofismo ante el dolor según el modelo de Sullivan et al. (1995), como paso previo a la traducción del instrumento. Este proceso se realizó de modo preciso y con la ayuda de expertos. En una segunda fase, el instrumento se administró a una muestra de deportistas con el objeto de examinar sus propiedades psicométricas. Tras este proceso, la escala ha quedado compuesta por 13 ítems (véase Anexo I).

Esta escala puede ser muy útil a la hora de valorar el estado psicológico de los deportistas cuando afrontan el dolor como consecuencia de una lesión y, por tanto, puede proporcionar información relevante para los profesionales encargados de supervisar y optimizar la rehabilitación de los atletas. Durante los últimos años, se ha demostrado la relación entre catastrofismo y dolor en diferentes poblaciones, por ejemplo, estudiantes universitarios (Sullivan et al., 1995), bailarinas (Encarnacion et al., 2001) o deportistas (Sullivan et al., 2000; Tripp et al., 2003). Además, los modelos psicológicos que explican el dolor desde una perspectiva cognitiva han mostrado que el pensamiento negativo puede dificultar la rehabilitación por el desequilibrio emocional derivado (Daly, Brewer, Van Raalte, Petitpas y Sklar, 1995), apoyando la idea de que controlar el catastrofismo en el deportista puede ayudar a disminuir los resultados negativos.

Se seleccionó la PCS por ser uno de los instrumentos más utilizados en todo el mundo para medir el catastrofismo ante el dolor y, aunque ha sido validado en población española (García-Campayo et al., 2008), ésta estaba formada por enfermos de fibromialgia. Sin embargo, no había sido utilizado en muestras de deportistas, población en la que el dolor, al cursar junto a la lesión deportiva, es un elemento cotidiano susceptible de ser analizado desde la perspectiva del catastrofismo. Una de las limitaciones del presente trabajo es que no compara la PCS con medidas de dolor percibido por los deportistas, tras ser expuestos a procedimientos dolorosos experimentales. Sin embargo, estos métodos fueron empleados por los autores originales cuando crearon la PCS (Sullivan et al., 1995) y no suelen utilizarse en validaciones a otro idioma.

Los resultados han mostrado las adecuadas propiedades psicométricas de la Escala de Catastrofismo ante el Dolor (ECD) que surge de la traducción y adaptación al español de la PCS en una muestra de deportistas españoles. Así, se mantiene la estructura original de tres factores descrita por Sullivan et al. (1995), confirmada por Sullivan et al. (2000), y ratificada en una muestra española por García-Campayo et al. (2008). La variancia explicada de cada uno de los factores (rumiación, 31.99\%; magnificación, 9.30\%; desesperación, $13.09 \%$ ) es similar a la descrita por Sullivan et al. (1995) en la escala original (un $41 \%$, un $10 \%$ y un $8 \%$, respectivamente) y la descrita por García-Campayo et al. (2008) (un 39\%, un $11 \%$ y un $10 \%$ ). En cuanto a la fiabilidad, los valores de consistencia interna (alfa de Cronbach), tanto de la escala como de dos de sus tres componentes son adecuados y superiores a 0,7 (excepto magnificación=0.618). El bajo coeficiente de consistencia interna para la subescala de magnificación puede reflejar la diversidad de situaciones de dolor que los individuos pueden haber considerado a la hora de cumplimentar el cuestionario. Tal y como explicaron Sullivan et al. (1995), el desarrollo de la PCS supuso que las situaciones de dolor comparten suficiente similitud como para provocar similares reacciones cognitivo-afectivas. Esta suposición puede valer más para la rumiación y la desesperación que para la magnificación. El número pequeño de ítems y el esfuerzo para minimizar la redundancia de los ítems pueden haber contribuido también al coeficiente de fiabilidad bajo. También es posible que los individuos que reflejan alguna forma de magnificación (ej. anticipando las consecuencias negativas) puedan no reflejar otras formas (ej. pensando en otras experiencias dolorosas), y así limitar la magnitud potencial de los coeficientes de fiabilidad.

Actualmente, no se han encontrado estudios sobre la correlación específica de cada una de las 3 subescalas de la PCS en deportistas. En el estudio realizado con bailarinas de $\mathrm{Pa}$ parizos et al. (2004), es la subescala de magnificación la que se correlaciona más estrechamente con la percepción de dolor y no el catastrofismo global como ocurría en la muestra de deportistas analizada por Sullivan et al. (2000). Otro caso relevante es el de los deportistas adolescentes intervenidos quirúrgicamente de ligamento cruzado anterior, que muestran índices superiores de rumiación y desesperación en comparación con sus homólogos adultos (Tripp et al., 2003). Por otra parte, en el estudio original de validación realizado en personas sanas (Sullivan et al., 1995), tanto la PCS global como la subescala de rumiación se correlacionaron mucho más intensamente con el dolor que con la depresión. En nuestro estudio con deportistas, la subescala de desesperación es la que mayor consistencia interna y mayor correlación de los ítems con la escala global presenta; sin embargo, es la rumiación la de mayor variancia explicada en consonancia con los resultados del estudio de García-Campayo et al. (2008) en pacientes de fibromialgia españoles.

$\mathrm{Al}$ analizar las correlaciones, tanto del catastrofismo global como de las tres subescalas en el grupo de los deportistas no lesionados, se apreció que no existe correlación ni con la ansie- 
dad rasgo ni con la depresión. A diferencia de los resultados de Sullivan et al. (1995) en la validación de la escala original, donde la PCS estaba correlacionada significativamente con la depresión y la ansiedad-rasgo en una muestra de estudiantes universitarios. Cuando se analizaron las relaciones del catastrofismo ante el dolor, y sus tres dimensiones, con la depresión y la ansiedad en el grupo de los deportistas lesionados, los resultados indican que no existen relaciones estadísticamente significativas con la depresión, salvo con la subescala de desesperación. Estos resultados discrepan con la investigación previa en diferentes poblaciones clínicas, donde el catastrofismo y la depresión estaban significativamente relacionados (Gracely et al., 2004; García-Campayo et al., 2008; Hasset, Cone, Patella y Sigal, 2000; Keefe et al., 2000; Sullivan et al., 1998; Severijns et al., 2002), lo que hace pensar que la depresión tiene un peso menor en el catastrofismo ante el dolor cuando se trata de deportistas lesionados. Sin embargo, en la revisión de Keefe et al. (2004) se hace referencia a numerosos estudios que avalan la relación entre la desesperación y la depresión, aunque los autores explican la desesperación como un constructo al margen del catastrofismo en los procesos de ajuste ante el dolor.

Por otro lado, sí se aprecian relaciones estadísticamente significativas entre la ECD global y sus tres subescalas, con la ansiedad rasgo, aunque los valores son bajos, posiblemente por el tamaño de la muestra. En este sentido, cabe destacar que la ansiedad y el catastrofismo ya han sido relacionados en estudios con deportistas como el de Tripp et al. (2003), realizado con adolescentes y adultos post-quirúrgicos; en po- blaciones clínicas (McCracken, Zayfert y Gross, 1992); así como en el estudio de validación de la PCS original (Sullivan et al., 1995). Por contra, los autores que han validado la versión española de la PCS en pacientes con fibromialgia (García-Campayo et al., 2008) hallaron que la correlación de la PCS con la ansiedad era relativamente baja, en línea con nuestro trabajo.

Por último, aunque la lesión es un obstáculo que aparece con mucha frecuencia en la carrera de los deportistas, los procesos cognitivos y emocionales que afectan a esta experiencia del atleta es un área poco investigada dentro del ámbito de la recuperación de las lesiones deportivas. Uno de los aspectos menos investigados es el papel del catastrofismo al dolor en deportistas lesionados y su impacto en la rehabilitación, quizá por falta de consenso en cuanto a su verdadera naturaleza, aunque tal y como indica Leung (2012) los recientes avances en genómica poblacional y de neuroimagen no invasiva están ayudado a conocer sus correlatos neurofisiológicos subyacentes, lo que anima a seguir en esta línea de investigación. Con la adaptación y validación de la ECD esperamos facilitar la realización de futuras investigaciones con deportistas espańoles lesionados.

En conclusión, este estudio confirma las adecuadas propiedades psicométricas de la ECD en deportistas españoles. Además, indica que en deportistas lesionados el catastrofismo ante el dolor se correlaciona más con la ansiedad que con la depresión, a diferencia de otros estudios realizados con muestras clínicas.

\section{Referencias bibliográficas}

1. Ahem, D.K. y Lohr, B.A. (1997). Psychosocial factors in sports injury rehabilitation. Clinics in Sports Medicine, 1, 755-767.

2. Beck, A.T. (1970). Depression: Causes and Treatment. Philadelphia, PA: University of Pennsylvania Press.

3. Beck, A.T., Ward, C.H., Mendelson, M., Mock, J. y Erbaugh, J. (1961). An inventory for measuring depression. Archives of General Psychiatry, 4, 561-571.

4. Buceta, J.M. (1996). Psicología y Lesiones Deportivas: Prevención y Recuperación. Madrid: Dykinson.

5. Bumberry, W., Oliver, J.M. y McClure, J.N. (1978). Validation of the Beck Depression Inventory in a university population using psychiatric estimate as the criterion. Journal of Consulting and Clinical Psychology, 46, 150-155.

6. Burns, J., Glenn, B., Bruehl, S., Harden, R. y Lofland, K. (2003). Cognitive factors influence outcome following multidisciplinary chronic pain treatment: A replication and extension of a cross-lagged panel analysis. Behavior Research and Therapy, 41, 1163-1182.

7. Burns, K., Kubilus, A., Bruehl, S., Harden, R. y Lofland, K. (2003). Do changes in cognitive factors influence outcome following multidisciplinary treatment for chronic pain? A cross-lagged panel analysis. Journal of Consulting and Clinical Psychology, 71, 81-91.

8. Butler, R.W., Damarin, F.L., Beaulieu, C., Schwebel, A.I. y Thorn BE. (1989). Assessing cognitive coping strategies for acute postsurgical pain. Journal of Consulting and Clinical Psychology, 1, 41-5.
9. Cano, A., Leonard, M.T. y Franz, A. (2005). The significant other version of the Pain Catastrophizing Scale (PCS-S): Preliminary validation. Pain, 119, 26-37.

10. Chibnall, J.T. y Tait, R.C. (2005). Confirmatory factor analysis of the pain catastrophizing scale in African American and Caucasian workers' compensation claimants with low back injuries. Pain, 113, 369-375.

11. Cramer, J.L. y Perna, F.M. (2000). Psychology/counselling: a universal competency in athletic training. Journal of Athletic Training, 35(4), 458-465.

12. Crombez, G., Bijttebier, P., Eccleston, C., Mascagni, T., Mertens, G., Goubert, L. y Verstraeten K. (2003). The child version of the Pain Catastrophizing Scale (PCS-C): a preliminary validation. Pain, 104, 639-646.

13. Crombez, G., Vlaeyen, J.W.S., Heuts, P.H.T.G. y Lysens, R. (1999). Pain related fear is more disabling than pain itself: Evidence on the role of pain-related fear in chronic back pain disability. Pain, 80, 329-339.

14. Daly, J.M., Brewer, B.W., Van Raalte, J.L., Petitpas, A.J. y Sklar, J.H. (1995). Cognitive appraisal, emotional adjustment, and adherence to rehabilitation following knee surgery. Journal of Sport Rehabilitation, 4, 22-30.

15. Edwards, R.R., Clifton, O., Bingham III, Bathon, J. y Haythornthwaite, J. (2006). Catastrophizing and Pain in Arthritis, Fibromyalgia, and Other Rheumatic Diseases. Arthritis and Rheumatism, 55(2), 325-332. 16. Edwards, R.R., Fillingim, R.B., Maixner, W., Sigurdsson, A. y Ha- 
ythornthwaite, J. (2004). Catastrophizing predicts changes in thermal pain responses after resolution of acute dental pain. Pain, 5, 164-170.

17. Edwards, R.R., Smith, M.T., Kudel, I. y Haythornthwaite J. (2006). Pain-related catastrophizing as a risk factor for suicidal ideation in chronic pain. Pain, 126, 272-279.

18. Encarnacion, M.L.G., Meyers, M.C., Ryan, N.D. y Pease, D.G. (2001). Pain coping styles of ballet performers. Journal of Sport Behavior, 23, 20-32.

19. García-Campayo, J., Rodero, B., Alda, M., Sobradiel, N., Montero, J. y Moreno, S. (2008). Validación de la versión espańola de la escala de catastrofización ante el dolor (Pain Catastrophizing Scale) en la fibromialgia. Medicina Clinica, 131(13), 487-492.

20. Geisser, M., Robinson, M., Keefe, F., Weiner, M., et al. (1994). Catastrophizing, depression and the sensory, affective and evaluative aspects of chronic pain. Pain, 59, 79-83.

21. Gracely, R.H., Geisser, M.E., Giesecke, T., Grant, M.A., Petzke, F., Williams D.A., et al. (2004). Pain catastrophizing and neural responses to pain among persons with fibromyalgia. Brain, 127, 835-843.

22. Haaga, D. (1990) Catastrophizing, confounds, and depression: A comment on Sullivan and D'Eon. Journal of Abnormal Psychology, 101, 206207.

23. Hamblenton, R.K. y Patsula, L. (1999). Increasing the validity of adapted test: Myths to be avoided and guidelines for improving test adaptation practices. Journal of Applied Testing Technology, 1. En http://www. testpublidhers.org/journal1.htm

24. Hamilton, L.H., Hamilton, W.G., Meitzer, J.D., Marshall. P. y Molnar, M. (1989). Personality, stress, and injuries in professional ballet dancers. The American Journal of Sports Medicine, 17, 263-261.

25. Hasset, A.L., Cone, J.D., Patella, S.J. y Sigal, L.H. (2000). The role of catstrophizing in the pain and depression of women with fibromialgia syndrome. Arthritis and Rheumatism, 43, 2493-2500.

26. Heil, J. (1993). Psychology of Sport Injury. Champaigne, Ill.: Human Kinetics.

27. Jensen, M., Ehde, D., Hoffman, A., Patterson, D., Czerniecki, J. y Robinson, L. (2002). Cognitions, coping and social environment predict adjustment to phantom limb pain. Pain, 95, 133-142.

28. Jensen, M., Turner, J., Romano, J., et al. (1991). Coping with chronic pain: A critical review of the literature. Pain, 47, 249-283

29. Keefe, F.J., Affleck, G., Lefebvre, J., Underwood, L., Caldwell, D.S., Drew, J., Egert, J. y Gibson, J. (2000). Living with rheumatoid arthritis: The role of daily spirituality and daily religious and spiritual coping. Journal of Pain, 2, 101-110.

30. Keefe, F.J., Rumble, M.E., Scipio, C.D., Giordano, L.A. y Perri, L.M. (2004). Psychological aspects of persistent pain: current state of the science. Journal of Pain, 5(4), 195-211.

31. Kendell, K., Saxby, B., Farrow, M. y Naisby, C. (2001). Psychological factors associated with short-term recovery from total knee replacement. British Journal of Health Psychology, 6, 41-52.

32. Leung, L. (2012). Pain catastrophizing: An updated review. Indian Journal of Psychological Medicine, 34, 204-217.

33. McCracken, L.M., Zayfert, C., y Gross, R.T. (1992). The Pain Anxiety Symptoms Scale: development and validation of a scale to measure fear of pain. Pain, 50, 67-73.

34. McWilliams, L.A. y Asmundson, G.J.G. (2007). The relationship of adult attachment dimensions to pain-related fear, hypervigilance, and catastrophizing. Pain, 127, 27-34.

35. Meyer, K., Sprott, H. y Mannion, A.F. (2008). Cross-cultural adaptation, reliability, and validity of the German version of the Pain Catastrophizing Scale. Journal of Psychosomatic Research, 64(5), 469-478.

36. Meyers, M.C., Bourgeois, A.E. y LeUnes, A. (2001). Pain coping response of collegiates athletes involved in high contact, high injurypotencial sport. International Journal of Sport Psychology, 32(1), 29-42.

37. Meyers, M.C., Bourgeois, A.E., Stewart, S. y LeUnes, A. (1992). Pre- dicting pain response in athletes: development and assessment of the Sport Inventory fon Pain. Journal of Sport and Exercise Psychology, 14(3), 249-261.

38. Miró, J., Nieto, R. y Huguet, A. (2008). The Catalan version of the Pain Catastrophizing Scale: a useful instrument to assess catastrophic thinking in whiplash patients. Journal of Pain, 9(5), 397-406.

39. Moral, J., González-Ramírez, M.T. y Landero, R. (2011). Evaluación de un tratamiento cognitivo-conductual para la fibromialgia. Ciencia UANL, 14(2), 153-161.

40. Neame, R. y Hammond, A. (2005). Beliefs about medications: a questionnaire survey of people with rheumatoid arthritis. Rheumatology, 44, 762-767.

41. Paparizos, A.I., Tripp, D.E., Sullivan, M.J.L. y Rubenstein, M.L. (2004). Catastrophizing and Pain Perception in Recreational Ballet Dancers. Journal of Sport Behavior, 28(1), 35-50.

42. Picavet, H.S., Vlaeyen, J.W. y Schouten, J.S. (2002). Pain catastrophizing and kinesiophobia: predictors of chronic low back pain. American Journal of Epidemiology, 156, 1028-1034.

43. Plata-Muńoz, M.A., Castillo-Olivares, M.A. y Guevara-López, U.M. (2004). Evaluación de afrontamiento, depresión, ansiedad e incapacidad funcional en pacientes con dolor crónico. Revista Mexicana de Anestesiologia, 27(1), 16-23.

44. Ramírez, A., Alzate, R. y Lázaro, I. (2002). Efectos psicológicos de la lesión deportiva. Motricidad, 9, 209-224.

45. Reiss, S. (1991). Expectancy theory of fear, anxiety and panic. Clinical Psychology Review, 11, 141-153.

46. Rodero, B., García-Campayo, J., Casanueva-Fernández, B. y Sobradiel, N. (2008). Imagined exposure as treatment of catastrophizing in fibromyalgia: a pilot study. Actas Españolas de Psiquiatría, 36(4), 223-226.

47. Rosenstiel, A. y Keefe, F. (1983). The use of coping strategies in chronic low back pain patients: Relationship to patient characteristics and $\mathrm{cu}-$ rrent adjustment. Pain, 17, 33-44.

48. Severeijns, R., Van den Hout, M.A., Vlaeyen, J.W. y Picavet, H.S (2002). Pain catastrophizing and general health status in a large Dutch community sample. Pain 99(1-2), 367-376.

49. Spielberger, C.D., Gorsuch, R.L. y Lushene, R.E. (1970). Manual for the State-Trait Anxiety Inventory. Palo Alto, CA: Consulting Psychologists Press.

50. Spielberger, C.D., Gorsuch, R.L., Lushene, R., Vagg, P.R. y Jacobs, G.A. (1983). State-Trait Anxiety Inventory for Adults: Sampler Set, Manual, Test, Scoring Key. Palo Alto, CA: Consulting Psychologists Press.

51. Stephens, M.A., Druley, J.A. y Zautra, A.J. (2002) .Older adults' recovery from surgery for osteoarthritis of the knee: psychosocial resources and constraints as predictors of outcomes. Health Psychology, 21, 377383.

52. Sullivan, M.J.L., Bishop, S.R. y Pivick, J. (1995). The pain catastrophizing scale: Development and Validation. Psychological Assessment, 7(4), 524-532.

53. Sullivan, M.J.L., Stanish, W., Waite, H., Sullivan, M. y Tripp, D. (1998). Catastrophizing, pain, and disability in patients with soft tissue injuries. Pain, 77, 253-260.

54. Sullivan, M.J.L., Thorn, B.E., Haythornthwaite, J.A., Keefe, F., Martin, M., Bradley, L. y Lefebvre, J.C. (2001). Theoretical perspectives on the relation between catastrophizing and pain. The Clinical Journal of Pain, 17, 52-64.

55. Sullivan, M.J.L., Tripp, D.A., Rodgers, W.M. y Stanish, W. (2000). Catastrophizing and pain perception in sport participants. Journal of Applied of Sport Psychology, 12, 151-167.

56. Tajet-Foxell, B. y Rose, D. (1995). Pain and pain tolerance in professional ballet dancers. British Journal of Sports Medicine, 29, 31-34.

57. Tremblay, I., Beaulieu, Y., Bernier, A., Crombez, G., Laliberté, S., y Sullivan, M.J.L. (2006). Adaptation et validation du «Pain Catastrophizing Scale» pour les adolescents francophones: impacts cliniques et 
scientifiques. Reproduction des appels de communications reçus pour le Carrefour des connaissances, p.10.

58. Tripp, D.A., Stanish, W.D., Ebel-Lam, A., Brewer, B.W. y Birchard, J. (2011). Fear of injury, negative affect, and catastrophizing predicting return to sport in recreational athletes with anterior cruciate ligament injuries at 1 year postsurgery. Sport, Exercise, and Performance Psycho$\log y, I(S), 38-48$.

59. Tripp, D.A., Stanish, W.D., Reardon, G., Coady, C., y Sullivan, M.J.L. (2003). Comparing postoperative pain experiences of the adolescent and adult athlete after anterior cruciate ligament surgery. Journal of Athletic Training, 38(2), 154-157.

60. Van Damme, S., Crombez, G., Bijttebier, P., Goubert, L., y Van Hou- denbove, B. (2002). A confirmatory factor analysis of the Pain Catastrophizing Scale: Invariant factor structure across clinical and non-clinical populations. Pain, 96(3), 319-324.

61. Wiese-Bjornstal, D.M (2002). Pain, no gain. Athletic Therapy Today, 7(5), 56-57.

62. Wiese-Bjornstal, D.M., Smith, A.M., Shaffer, S.M., y Morrey, M.A (1998). An integrated model of response to sport injury: Psychological and sociological dynamics. Journal of Applied Sport Psychology, 10, 46-69.

63. Yap, J.C., Lau, J., Chen, P.P., Gin, T., Wong, T., Chan, I., Chu, J., y Wong, E. (2008). Validation of the Chinese Pain Catastrophizing Scale (HK-PCS) in patients with chronic pain. Pain Medicine, 9(2), 186-195. 


\section{Anexo}

\section{ESCALA DE CATASTROFISMO ANTE EL DOLOR (ECD)}

Todos los deportistas experimentan situaciones de dolor en algún momento de su carrera deportiva; en muchas ocasiones estas situaciones de dolor están relacionadas con las lesiones sufridas. Esta escala trata de conocer los pensamientos y los sentimientos de los deportistas cuando experimentan dolor.

Todos los datos e información obtenida serán tratados de forma anónima, sirviendo únicamente al objeto de la investigación para la que van dirigidos.

\begin{tabular}{|l|l|l|l|}
\hline Deporte & Sexo & \\
\hline Categoría competición & Edad & \\
\hline Años federado & Puesto en el que juega & \\
\hline Si actualmente se encuentra lesionado, por favor, cumplimente estas dos cuestiones & \\
\hline ¿Qué lesión tiene? & Tiempo de recuperación estimado & \\
\hline
\end{tabular}

Por favor, lea con atención y complete con sinceridad marcando de forma clara (con una "X”) la opción elegida en cada una de las 13 afirmaciones.

Tomando como referencia la experiencia de dolor presente en su carrera deportiva, indique el grado en el cual experimenta cada uno de los pensamientos o los sentimientos en una escala de 0 (nunca) a 4 (siempre).

Cuando siento dolor...

\begin{tabular}{ll}
\hline SITUACIÓN DE DOLOR & NUNCA RARA VEZ $\begin{array}{c}\text { ALGUNAS MUCHAS } \\
\text { VECES }\end{array}$ \\
\hline
\end{tabular}

1. Me preocupo sobre si el dolor se acabará.

2. Siento que ya no puedo continuar debido al dolor.

3. El dolor es muy fuerte y creo que nunca va a mejorar.

4. El dolor es muy desagradable y siento que me supera.

5. Siento que no aguanto más el dolor.

6. Tengo miedo de que el dolor pueda ir en aumento.

7. Me vienen a la memoria experiencias dolorosas anteriores.

8. Deseo con muchas ganas que el dolor desaparezca.

9. No paro de pensar en el dolor.

10. Estoy centrado en cuanto me duele.

11. Pienso en que lo quiero es que me deje de doler.

12. No puedo hacer nada para disminuir la intensidad del dolor.

13. Me pregunto si me podría pasar algo grave.

\begin{tabular}{lllll}
0 & 1 & 2 & 3 & 4 \\
0 & 1 & 2 & 3 & 4 \\
0 & 1 & 2 & 3 & 4 \\
0 & 1 & 2 & 3 & 4 \\
0 & 1 & 2 & 3 & 4 \\
0 & 1 & 2 & 3 & 4 \\
0 & 1 & 2 & 3 & 4 \\
0 & 1 & 2 & 3 & 4 \\
0 & 1 & 2 & 3 & 4 \\
0 & 1 & 2 & 3 & 4 \\
0 & 1 & 2 & 3 & 4 \\
0 & 1 & 2 & 3 & 4 \\
0 & 1 & 2 & 3 & 4 \\
\hline
\end{tabular}

Agradecemos su colaboración. 